\title{
Revisiting secondary normal pressure hydrocephalus: does it exist? A review
}

\author{
Badih Daou, MD,1 Petra Klinge, MD, ${ }^{2}$ Stavropoula Tjoumakaris, MD, ${ }^{1}$ Robert H. Rosenwasser, MD, ${ }^{1}$ \\ and Pascal Jabbour, MD'
}

\begin{abstract}
1'Department of Neurological Surgery, Thomas Jefferson University and Jefferson Hospital for Neuroscience, Philadelphia, Pennsylvania; and 'Department of Neurosurgery, The Warren Alpert Medical School of Brown University, Rhode Island Hospital, Providence, Rhode Island
\end{abstract}

OBJECTIVE There are several etiologies that can lead to the development of secondary normal pressure hydrocephalus (sNPH). The aim of this study was to evaluate the etiology, diagnosis, treatment, and outcome in patients with sNPH and to highlight important differences between the separate etiologies.

METHODS A comprehensive review of the literature was performed to identify studies conducted between 1965 and 2015 that included data regarding the etiology, treatment, diagnosis, and outcome in patients with sNPH. Sixty-four studies with a total of 1309 patients were included. The inclusion criteria of this study were articles that were written in English, included more than 2 patients with the diagnosis of sNPH, and contained data regarding the etiology, diagnosis, treatment, or outcome of NPH. The most common assessment of clinical improvement was based on the Stein and Langfitt grading scale or equivalent improvement on other alternative ordinal grading scales.

RESULTS The main etiologies of sNPH were subarachnoid hemorrhage (SAH) in $46.5 \%$, head trauma in $29 \%$, intracranial malignancies in $6.2 \%$, meningoencephalitis in $5 \%$, and cerebrovascular disease in $4.5 \%$ of patients. In $71.9 \%$ of patients the sNPH was treated with ventriculoperitoneal shunt placement, and $24.4 \%$ had placement of a ventriculoatrial shunt. Clinical improvement after shunt placement was reported in $74.4 \%$ and excellent clinical improvement in $58 \%$ of patients with sNPH. The mean follow-up period after shunt placement was 13 months. Improvement was seen in $84.2 \%$ of patients with $\mathrm{SAH}, 83 \%$ of patients with head trauma, $86.4 \%$ of patients with brain tumors, $75 \%$ of patients with meningoencephalitis, and $64.7 \%$ of patients with NPH secondary to stroke.

CONCLUSIONS Secondary NPH encompasses a diverse group of clinical manifestations associated with a subset of patients with acquired hydrocephalus. The most common etiologies of sNPH include SAH and traumatic brain injury. Secondary NPH does indeed exist, and should be differentiated from idiopathic NPH based on outcome and on clinical, pathophysiological, and epidemiological characteristics, but should not be considered as a separate entity.

http://thejns.org/doi/abs/10.3171/2016.6.FOCUS16189

KEY WORDS normal pressure hydrocephalus; NPH; secondary

I N 1965, Hakim and Adams described a syndrome of symptomatic hydrocephalus with a normal CSF pressure that presents with gait disturbance, dementia, and incontinence without overt signs and symptoms of elevated intracranial pressure (ICP). ${ }^{17}$ They called it nor- mal pressure hydrocephalus (NPH) and found that it was a "treatable syndrome" with the performance of neurosurgical shunting procedures. ${ }^{1}$ Further studies have confirmed these findings and have shown that in spite of a normal CSF pressure approximately $50 \%$ of patients with NPH

ABBREVIATIONS CVD = cerebrovascular disease; $I \mathrm{CH}=$ intracerebral hemorrhage; ICP = intracranial pressure; $\mathrm{LP}=\mathrm{lumboperitoneal;} \mathrm{NPH}$, iNPH, $\mathrm{sNPH}=$ normal pressure hydrocephalus, idiopathic NPH, secondary NPH; SAH = subarachnoid hemorrhage; VA = ventriculoatrial; VP = ventriculoperitoneal.

SUBMITTED April 29, 2016. ACCEPTED June 29, 2016.

INCLUDE WHEN CITING DOI: 10.3171/2016.6.FOCUS16189. 
improve after shunt surgery. ${ }^{72}$ In $50 \%$ of patients with $\mathrm{NPH}$, no known cause can be identified. ${ }^{6}$ These cases are referred to as idiopathic NPH (iNPH). The other 50\% of $\mathrm{NPH}$ cases occur in association with prior events that lead to the development of this syndrome. These are termed secondary NPH (sNPH). The causes of sNPH are multiple and include mainly subarachnoid hemorrhage (SAH), trauma, malignancy, meningitis, stroke, and intracerebral hemorrhage (ICH). ${ }^{4}$ Idiopathic NPH generally occurs in adults during the 6th and 7th decades of life, whereas sNPH can occur at any age. ${ }^{20}$ Differences in outcome between iNPH and sNPH have been observed, with substantial improvement after shunt placement occurring in approximately $30 \%-50 \%$ of patients with iNPH and in approximately $50 \%-70 \%$ of patients with sNPH..$^{72}$

Many articles have described the diagnosis and management of NPH and the predictive tests used for selecting the most appropriate candidates for shunt placement. In 2005, Marmarou and colleagues published guidelines for the diagnosis and management of iNPH and emphasized the importance of distinguishing between iNPH and sNPH. $3,28,34,35,55$ Because sNPH is so heterogeneous with regard to pathology, diagnosis, treatment, and outcome, it is not clear if the term "secondary normal pressure hydrocephalus" should be used to encompass such a wide array of entities and whether unifying guidelines should be made for the diagnosis and management of sNPH. The aim of this review study was to evaluate the etiology, diagnosis, treatment, and outcome in patients with sNPH and to highlight important differences among the separate etiologies. We also sought to answer the following questions: What are the most common etiologies of sNPH? What are the treatments used to manage it? How successful are the treatments?

\section{Methods}

A comprehensive review of the literature was performed using the key words "secondary normal pressure hydrocephalus," "normal pressure hydrocephalus," "NPH," "hemorrhage and normal pressure hydrocephalus," "trauma and normal pressure hydrocephalus," "meningitis and normal pressure hydrocephalus," "tumor and normal pressure hydrocephalus," and "stroke and normal pressure hydrocephalus." These terms were used to conduct searches in the following databases: PubMed, Ovid, and Scopus. The databases were last queried on July 1,2015. The inclusion criteria of this study were articles published between 1965 and 2015, written in English, including more than 2 patients with the diagnosis of sNPH, and containing data regarding the etiology, diagnosis, treatment, or outcome of NPH. The exclusion criteria were the following: case reports, technical notes, review articles, and articles not written in English. Studies that included sNPH cases but did not elaborate on etiology, treatment, or outcome were excluded. Despite the methodological limitations, all publications meeting these criteria were included due to the paucity of research papers dealing with the topic of sNPH. Data regarding etiology, treatment, and outcome in patients with sNPH were extracted independently after a thorough review of the results of each published article.
Summary measures included assessment of the frequency of patients with certain etiologies and the mean number of patients who improved after shunt placement.

Most identified publications combined both iNPH and sNPH, or excluded sNPH cases. Many of these studies did not differentiate among diagnostic, treatment, and outcome differences between these 2 entities. The design, patient selection criteria, outcome criteria, and conclusions of each study were analyzed. Outcome definitions and postoperative follow-up periods varied widely across studies. Several scales and scores were used to assess clinical improvement following shunt placement. The most common assessment of clinical improvement was defined as a decrease of at least 1 grade on the Stein and Langfitt grading scale, or equivalent improvement on other ordinal grading scales. ${ }^{61}$ Marked improvement was defined as a decrease of at least 2 grades on the Stein and Langfitt grading scale, or equivalent improvement on other ordinal grading scales.

\section{Results}

\section{Study Selection}

The search generated approximately 3000 articles, of which 64 met our inclusion criteria (Table 1). 2.5,7-11,14,16,18,19, 21-24,26,27,29-33,36,38-50,52,54,56-63,65-71,73-83 A total of 1309 patients with sNPH were included. The mean number of patients per study was 21 . Of the articles that mentioned the study design, 21 were prospective and 27 were retrospective. Fifty-nine studies reported the etiology of sNPH, 61 studies reported the diagnostic tests used to identify patients with NPH and the prognostic tests used to predict the outcomes of shunt placement, 46 studies reported the type of shunt used for management of NPH cases, and 38 studies mentioned the valve system used. The outcome specific to patients with sNPH after shunt surgery was reported in 35 studies.

\section{Etiology of sNPH}

The etiology of sNPH was reported in 1208 patients (Table 2). The most common cause of $\mathrm{sNPH}$ was $\mathrm{SAH}(\mathrm{n}=$ $562,46.5 \%)$. The second most common etiology resulting in sNPH was head trauma $(n=349,29 \%)$. Other important etiologies included brain tumors and surgery for resection of intracranial malignancies in 75 patients $(6.2 \%)$, meningoencephalitis in 61 patients $(5 \%)$, cerebrovascular disease (CVD) in 55 patients (4.5\%), and ICH in 49 patients (4\%). Other etiologies made up for $5 \%$ of the causes of sNPH. These included mainly intracranial operations, radiosurgery, aqueductal stenosis, and Paget's disease.

\section{Diagnosis of sNPH}

The diagnosis of sNPH was based on a combination of clinical history, physical examination, and imaging studies. The NPH was considered to be secondary when an identifiable event occurred prior to the onset of symptoms and was judged to be directly related to the development of NPH. In most studies the diagnosis of NPH was established when patients presented with the classic symptoms of dementia, gait disturbance, and urinary incontinence accompanied by enlarged ventricles on brain imaging, with an Evans index of $\geq 0.3$ (maximum width of the 
TABLE 1. Evaluation of studies included in the literature review of patients with NPH

\begin{tabular}{|c|c|c|c|c|c|c|}
\hline Authors \& Year & $\begin{array}{l}\text { Study } \\
\text { Design }\end{array}$ & $\begin{array}{l}\text { No. } \\
\text { of Pts } \\
\text { W/ } \\
\text { sNPH }\end{array}$ & Etiology & $\begin{array}{l}\text { Follow-Up } \\
\text { Period }\end{array}$ & $\begin{array}{l}\text { Shunt } \\
\text { Treatment }\end{array}$ & Outcome \\
\hline $\begin{array}{l}\text { McQuarrie et al., } \\
1984\end{array}$ & $\begin{array}{l}\text { Retrospec- } \\
\quad \text { tive }\end{array}$ & 25 & SAH \& trauma: 25 & 3 mos & $\begin{array}{l}21 \text { VP \& } 4 \\
\text { VA shunts }\end{array}$ & $\begin{array}{l}53 \% \text { w/ early disease improved, whereas } 27 \% \text { w/ advanced } \\
\text { disease improved. }\end{array}$ \\
\hline $\begin{array}{l}\text { Reinprecht et } \\
\quad \text { al., } 1995\end{array}$ & $\begin{array}{l}\text { Retrospec- } \\
\text { tive }\end{array}$ & 20 & $\begin{array}{l}\text { SAH: } 12 ; \text { trauma: } 3 \\
\quad \text { tumor: } 5\end{array}$ & $7-29$ mos & $\begin{array}{l}\text { VP \& VA } \\
\text { shunts }\end{array}$ & $\begin{array}{l}71.1 \% \text { had complete reversal of symptoms \& } 21.1 \% \text { of pts } \\
\text { had improvement w/ residual symptoms (mixed iNPH \& } \\
\text { sNPH cases). }\end{array}$ \\
\hline Salmon, 1972 & $\begin{array}{l}\text { Retrospec- } \\
\text { tive }\end{array}$ & 44 & $\begin{array}{l}\text { SAH: } 3 \text {; trauma: } 26 \\
\text { meningoencephali- } \\
\text { tis: } 2 \text {; other: } 13\end{array}$ & 6 mos & VP shunts & $\begin{array}{l}9 \text { pts had moderate or marked improvement }(20 \%), 8 \\
\text { pts showed minimal improvement }(17.8 \%), 24 \text { had no } \\
\text { change }(53.3 \%), \& 4 \text { deteriorated }(8.9 \%) \text {. }\end{array}$ \\
\hline $\begin{array}{l}\text { Stein \& Langfitt, } \\
\quad 1974\end{array}$ & $\begin{array}{l}\text { Prospec- } \\
\text { tive }\end{array}$ & 10 & $\begin{array}{l}\text { SAH: } 3 \text {; trauma: } 3 \text {; } \\
\text { tumor: } 3 \text {; meningo- } \\
\text { encephalitis: } 1\end{array}$ & $\begin{array}{l}18 \text { mos } \\
\text { (mean) }\end{array}$ & Not specified & $\begin{array}{l}80 \% \text { w/ known etiology significantly improved. In pts w/ } \\
\text { iNPH, } 64 \% \text { showed some improvement; this was sub- } \\
\text { stantial \& sustained in } 24 \% \text {. }\end{array}$ \\
\hline $\begin{array}{l}\text { Vanneste et al., } \\
\quad 1993\end{array}$ & $\begin{array}{l}\text { Retrospec- } \\
\quad \text { tive }\end{array}$ & 23 & Not specified & $12 \mathrm{mos}$ & Not specified & $\begin{array}{l}56.5 \% \text { of sNPH cases had substantial improvement } \& 8.7 \% \\
\text { had some improvement. } 14.6 \% \text { of iNPH cases had sub- } \\
\text { stantial improvement \& } 15.9 \% \text { had some improvement. }\end{array}$ \\
\hline $\begin{array}{l}\text { Vanneste et al., } \\
\quad 1992\end{array}$ & $\begin{array}{l}\text { Retrospec- } \\
\text { tive }\end{array}$ & 25 & Not specified & $\begin{array}{l}3.1 \text { yrs } \\
\text { (me- } \\
\text { dian) }\end{array}$ & $\begin{array}{l}\text { VP \& VA } \\
\text { shunts }\end{array}$ & $\begin{array}{l}52 \% \text { of pts w/ sNPH had marked improvement \& } 8 \% \\
\text { had slight improvement. } 14.9 \% \mathrm{w} / \text { iNPH had marked } \\
\text { improvement \& } 16.5 \% \text { had slight improvement. }\end{array}$ \\
\hline $\begin{array}{l}\text { Walchenbach et } \\
\text { al., } 2002\end{array}$ & $\begin{array}{l}\text { Prospec- } \\
\text { tive }\end{array}$ & 6 & Not specified & $\begin{array}{l}2,6, \& 12 \\
\text { mos }\end{array}$ & VP shunts & $\begin{array}{l}\text { Clinically meaningful improvement after } 2 \text { mos was seen in } \\
73 \% \text { of pts. }\end{array}$ \\
\hline $\begin{array}{l}\text { Yamashita et al., } \\
1999\end{array}$ & $\begin{array}{l}\text { Retrospec- } \\
\text { tive }\end{array}$ & 65 & SAH: 65 & $\begin{array}{r}13.3 \text { mos } \\
\text { (mean) }\end{array}$ & VP shunt & $\begin{array}{l}\text { Shunt reprogramming was frequently performed in pts w/ } \\
\text { subdural effusion, meningitis, \& iNPH, but infrequently in } \\
\text { pts w/ sNPH after SAH. }\end{array}$ \\
\hline $\begin{array}{l}\text { Zemack \& Rom- } \\
\text { ner, } 2002\end{array}$ & $\begin{array}{l}\text { Retrospec- } \\
\text { tive }\end{array}$ & 71 & $\begin{array}{l}\text { SAH: } 37 \text {; trauma: } 21 \text {; } \\
\text { tumor: } 4 \text {; menin- } \\
\text { goencephalitis: } 2 \text {; } \\
\text { other: } 7\end{array}$ & $\begin{array}{r}26.7 \text { mos } \\
\text { (mean) }\end{array}$ & $\begin{array}{l}\text { VP }(89.5 \%) \\
\quad \& \text { VA } \\
(10.5 \%) \\
\text { shunts }\end{array}$ & $\begin{array}{l}\text { Outcomes were excellent or good in } 69.8 \% \text { of pts w/ sNPH } \\
\text { \& in } 78.9 \% \text { of pts w/ iNPH. }\end{array}$ \\
\hline $\begin{array}{l}\text { Kahlon et al., } \\
2002\end{array}$ & $\begin{array}{l}\text { Prospec- } \\
\quad \text { tive }\end{array}$ & 17 & $\begin{array}{l}\text { SAH: } 1 \text {; trauma: } 5 \text {; } \\
\text { tumor: } 2 \text {; menin- } \\
\text { goencephalitis: } 2 \text {; } \\
\text { ICH: } 7\end{array}$ & $\begin{array}{l}6 \text { mos } \\
\text { (mean) }\end{array}$ & $\begin{array}{l}\text { VP or } \\
\text { ventricu- } \\
\text { lovenous } \\
\text { shunt }\end{array}$ & Postop assessments verified improvements in $81 \%$ of pts. \\
\hline $\begin{array}{l}\text { Larsson et al., } \\
\quad 1991\end{array}$ & $\begin{array}{l}\text { Retrospec- } \\
\quad \text { tive }\end{array}$ & 48 & $\begin{array}{l}\text { SAH: } 19 \text {; trauma: } 15 \\
\text { tumor: } 3 \text {; CVD: } 11\end{array}$ & $\begin{array}{l}2.1 \mathrm{yrs} \\
\text { (mean) }\end{array}$ & VP shunts & $\begin{array}{l}\text { Improvement occurred in } 78 \% \text {, \& } 22 \% \text { deteriorated. Im- } \\
\text { provement in clinical function was seen in } 94 \% \text { of SAH } \\
\text { pts, } 79 \% \text { of posttrauma pts, } 77 \% \text { of iNPH pts, \& } 70 \% \text { of } \\
\text { pts w/ CVD. }\end{array}$ \\
\hline Magnaes, 1978 & $\begin{array}{l}\text { Retrospec- } \\
\text { tive }\end{array}$ & 34 & $\begin{array}{l}\text { SAH: } 14 ; \text { trauma: } 8 \\
\text { tumor: } 7 ; \mathrm{ICH}: 5\end{array}$ & $\begin{array}{r}3 \& 12 \\
\text { mos }\end{array}$ & VA shunts & $59.2 \%$ of sNPH \& $33.3 \%$ of iNPH cases improved. \\
\hline $\begin{array}{l}\text { Gustafson \& } \\
\text { Hagberg, } \\
1978\end{array}$ & $\begin{array}{l}\text { Prospec- } \\
\text { tive }\end{array}$ & 18 & $\begin{array}{l}\text { SAH: } 5 \text {; trauma: } 7 \\
\text { tumor: } 2 \text {; menin- } \\
\text { goencephalitis: } 1 \text {; } \\
\text { other: } 3\end{array}$ & $3-6 \mathrm{mos}$ & VA shunts & $50 \%$ of pts improved. \\
\hline $\begin{array}{l}\text { Thomsen et al., } \\
1986\end{array}$ & $\begin{array}{l}\text { Prospec- } \\
\quad \text { tive }\end{array}$ & 21 & $\begin{array}{l}\text { SAH: } 9 \text {; trauma: } 9 \text {; } \\
\text { other: } 3\end{array}$ & $\begin{array}{r}3 \& 12 \\
\text { mos }\end{array}$ & VA shunts & $14 / 21$ pts w/ sNPH improved vs 2/19 pts w/ iNPH. \\
\hline $\begin{array}{l}\text { Mathew et al., } \\
1975\end{array}$ & $\begin{array}{l}\text { Prospec- } \\
\text { tive }\end{array}$ & 10 & $\begin{array}{l}\text { SAH: } 7 \text {; trauma: } 1 \text {; } \\
\text { meningoencephali- } \\
\text { tis: } 1 ; \text { CVD: } 1\end{array}$ & $6 \mathrm{mos}$ & $\begin{array}{l}\text { VA \& VP } \\
\text { shunts }\end{array}$ & $\begin{array}{l}\text { Excellent outcome: } 3 \text { sNPH cases ( } 1 \text { trauma, } 1 \text { SAH, } 1 \\
\text { meningitis) \& } 1 \text { iNPH case. Good outcome: } 2 \text { sNPH } \\
\text { (after SAH) \& } 0 \text { iNPH cases. Fair outcome: } 3 \text { sNPH \& } 2 \\
\text { iNPH cases. Poor outcome: } 2 \text { sNPH \& } 2 \text { iNPH cases. }\end{array}$ \\
\hline $\begin{array}{l}\text { Wood et al., } \\
1974\end{array}$ & $\begin{array}{l}\text { Retrospec- } \\
\text { tive }\end{array}$ & 46 & $\begin{array}{l}\text { SAH: } 16 \text {; trauma: } 14 \text {; } \\
\text { meningoencepha- } \\
\text { litis: } 4 \text {; CVD: } 8 \text {; } \\
\text { other: } 4\end{array}$ & $1-7$ yrs & $\begin{array}{l}\text { VA, VP, or } \\
\text { theco- } \\
\text { peritoneal } \\
\text { shunts }\end{array}$ & $60 \%$ improved. \\
\hline
\end{tabular}


TABLE 1. Evaluation of studies included in the literature review of patients with NPH

\begin{tabular}{|c|c|c|c|c|c|c|}
\hline Authors \& Year & $\begin{array}{l}\text { Study } \\
\text { Design }\end{array}$ & $\begin{array}{l}\text { No. } \\
\text { of Pts } \\
\text { W/ } \\
\text { sNPH }\end{array}$ & Etiology & $\begin{array}{l}\text { Follow-Up } \\
\text { Period }\end{array}$ & $\begin{array}{l}\text { Shunt } \\
\text { Treatment }\end{array}$ & Outcome \\
\hline $\begin{array}{l}\text { Ishikawa et al., } \\
1989\end{array}$ & $\begin{array}{l}\text { Retrospec- } \\
\text { tive }\end{array}$ & 6 & $\mathrm{SAH}: 6$ & $\begin{array}{l}\text { Not speci- } \\
\text { fied }\end{array}$ & Not specified & Excellent outcome in all 6 pts (100\%) w/ sNPH. \\
\hline $\begin{array}{l}\text { Chen et al., } \\
1994\end{array}$ & $\begin{array}{l}\text { Retrospec- } \\
\text { tive }\end{array}$ & 31 & $\begin{array}{l}\text { SAH: } 4 \text {; trauma: } 20 \text {; } \\
\quad \mathrm{ICH}: 7\end{array}$ & $6 \mathrm{mos}$ & VP shunts & $71 \%$ of pts had improvement. \\
\hline $\begin{array}{l}\text { Chen et al., } \\
2009\end{array}$ & $\begin{array}{l}\text { Retrospec- } \\
\text { tive }\end{array}$ & 39 & SAH: 39 & $1 \& 6$ mos & $\begin{array}{l}\text { VP shunts in } \\
61.5 \% \text { \& } \\
\text { no shunt } \\
\text { in } 38.5 \%\end{array}$ & $\begin{array}{l}\text { There was a significant improvement in pts who had CSF } \\
\text { shunting as compared to pts w/o shunt treatment. }\end{array}$ \\
\hline Wen et al., 2009 & $\begin{array}{l}\text { Retrospec- } \\
\text { tive }\end{array}$ & 31 & Trauma: 31 & $12 \mathrm{mos}$ & Not specified & $\begin{array}{l}64.5 \% \text { showed clear improvement, \& the other pts re- } \\
\text { mained unchanged or deteriorated. }\end{array}$ \\
\hline $\begin{array}{l}\text { Eide \& Sorte- } \\
\text { berg, } 2008\end{array}$ & $\begin{array}{l}\text { Retrospec- } \\
\text { tive }\end{array}$ & 4 & SAH: 4 ; trauma: 1 & 3 mos & VP shunts & All sNPH pts had improvement in NPH score. \\
\hline $\begin{array}{l}\text { Pfisterer et al., } \\
2007\end{array}$ & $\begin{array}{l}\text { Prospec- } \\
\text { tive }\end{array}$ & 18 & $\begin{array}{l}\text { SAH: } 5 \text {; trauma: } 8 \\
\text { meningoencepha- } \\
\text { litis: } 5\end{array}$ & $\begin{array}{l}6.5 \text { yrs } \\
\text { (me- } \\
\text { dian) }\end{array}$ & VA shunts & $\begin{array}{l}96.1 \% \text { improved in gait disturbance, } 77.1 \% \text { in cognitive } \\
\text { impairment, \& } 75.7 \% \text { in urinary dysfunction. }\end{array}$ \\
\hline Kilic et al., 2007 & $\begin{array}{l}\text { Retrospec- } \\
\text { tive }\end{array}$ & 49 & $\begin{array}{l}\text { Trauma: } 26 \text {; menin- } \\
\text { goencephalitis: } 8 \text {; } \\
\text { ICH: } 15\end{array}$ & $\begin{array}{l}25 \text { mos } \\
\quad \text { (mean) }\end{array}$ & $\begin{array}{l}\text { VP \& VA } \\
\text { shunts }\end{array}$ & $\begin{array}{l}\text { Improvement observed in } 88 \% \text { of pts who had CSF tap } \\
\text { test, } 91 \% \text { of pts who had external lumbar drainage, } \& \\
66 \% \text { of pts who had cisternography. }\end{array}$ \\
\hline $\begin{array}{l}\text { Chang et al., } \\
1999\end{array}$ & $\begin{array}{l}\text { Retrospec- } \\
\text { tive }\end{array}$ & 26 & $\begin{array}{l}\text { SAH: 18; trauma: } 7 \\
\text { ICH: } 1\end{array}$ & $\begin{array}{l}\text { Not speci- } \\
\text { fied }\end{array}$ & LP shunts & $30 / 32$ pts improved after surgery $(93.75 \%)$ \\
\hline $\begin{array}{l}\text { Panagiotopoulos } \\
\text { et al., } 2005\end{array}$ & $\begin{array}{l}\text { Prospec- } \\
\text { tive }\end{array}$ & 3 & Not specified & $3 \mathrm{mos}$ & VP shunts & NA \\
\hline Poca et al., 2005 & $\begin{array}{l}\text { Retrospec- } \\
\text { tive }\end{array}$ & 13 & $\begin{array}{l}\text { SAH: } 2 \text {; tumor: } 3 \text {; me- } \\
\text { ningoencephalitis: } \\
\text { 2; ICH: } 1 \text {; other: } 5\end{array}$ & $3 \mathrm{mos}$ & Not specified & NA \\
\hline $\begin{array}{l}\text { Owler et al., } \\
2004\end{array}$ & $\begin{array}{l}\text { Not speci- } \\
\text { fied }\end{array}$ & 5 & $\begin{array}{r}\text { Tumor: } 1 \text {; meningoen- } \\
\text { cephalitis: } 2 ; \mathrm{ICH}: 1\end{array}$ & $\begin{array}{l}\text { Not speci- } \\
\quad \text { fied }\end{array}$ & VP shunts & NA \\
\hline $\begin{array}{l}\text { Chang et al., } \\
1999\end{array}$ & $\begin{array}{l}\text { Prospec- } \\
\text { tive }\end{array}$ & 28 & $\begin{array}{l}\text { SAH: } 14 ; \text { trauma: } 10 \\
\text { tumor: } 1 ; \mathrm{ICH}: 3\end{array}$ & $3 \mathrm{mos}$ & $\begin{array}{l}\text { VP \& LP } \\
\text { shunts }\end{array}$ & $\begin{array}{l}\text { Excellent \& good outcomes were seen in } 64.3 \% \text { of pts w/ } \\
\text { sNPH \& } 22.2 \% \text { of pts w/ iNPH. }\end{array}$ \\
\hline Lee et al., 2012 & $\begin{array}{l}\text { Prospec- } \\
\text { tive }\end{array}$ & 9 & $\begin{array}{l}\text { SAH: } 7 \text {; trauma: } 1 \text {; aq- } \\
\text { ueductal stenosis: } 1\end{array}$ & $3 \mathrm{mos}$ & Not specified & 7 pts responded, 4 pts had shunt failure. \\
\hline $\begin{array}{l}\text { Shiino et al., } \\
2004\end{array}$ & $\begin{array}{l}\text { Prospec- } \\
\text { tive }\end{array}$ & 21 & $\begin{array}{l}\text { SAH: 17; tumor: } 1 \text {; me- } \\
\text { ningoencephalitis: } \\
\text { 2; ICH: } 1\end{array}$ & $\begin{array}{r}1 \& 12 \\
\text { mos }\end{array}$ & VP shunts & Outcome was excellent in 10 pts, fair in $5, \&$ poor in 4. \\
\hline $\begin{array}{l}\text { Kosteljanetz et } \\
\quad \text { al., } 1990\end{array}$ & $\begin{array}{l}\text { Prospec- } \\
\text { tive }\end{array}$ & 8 & $\begin{array}{l}\text { SAH: } 5 \text {; trauma: } 1 \text {; } \\
\text { meningoencephali- } \\
\text { tis: } 1 ; \text { CVD: } 1\end{array}$ & 3 mos & VP shunt & $\begin{array}{l}\text { 2/4 pts w/ SAH who underwent CSF shunting improved. } \\
3 / 9 \text { pts w/ iNPH who underwent CSF shunting improved. }\end{array}$ \\
\hline $\begin{array}{l}\text { Chen et al., } \\
\quad 1994\end{array}$ & $\begin{array}{l}\text { Retrospec- } \\
\text { tive }\end{array}$ & 8 & Trauma: 5; CVD: 3 & $12 \mathrm{mos}$ & $\begin{array}{l}\text { VP \& LP } \\
\text { shunts }\end{array}$ & 8 pts had improvement. \\
\hline $\begin{array}{l}\text { Hashimoto et al., } \\
\qquad 1990\end{array}$ & $\begin{array}{l}\text { Prospec- } \\
\text { tive }\end{array}$ & 15 & $\begin{array}{l}\text { SAH: } 13 \text {; trauma: } 1 \text {; } \\
\quad I C H: 1\end{array}$ & 2 mos & VP shunts & Shunt was ineffective in 7 pts $\&$ was effective in 8. \\
\hline $\begin{array}{l}\text { Wikkelsö et al., } \\
\quad 1986\end{array}$ & $\begin{array}{l}\text { Prospec- } \\
\text { tive }\end{array}$ & 21 & $\begin{array}{l}\text { SAH: } 9 ; \text { trauma: } 8 \\
\text { CVD: } 4\end{array}$ & $3-6$ mos & VP shunts & $\begin{array}{l}\text { 9/9 pts w/ NPH after SAH had excellent improvement. } 3 / 8 \\
\text { w/ head trauma did not improve, } 4 \text { improved, \& only } 1 \\
\text { showed excellent improvement. } 3 \text { pts w/ iNPH improved, } \\
\& 2 \text { were unchanged. } 2 / 2 \text { pts w/ CVA who received } \\
\text { follow-up improved. }\end{array}$ \\
\hline $\begin{array}{l}\text { Vorstrup et al., } \\
\quad 1987\end{array}$ & $\begin{array}{l}\text { Not speci- } \\
\quad \text { fied }\end{array}$ & 3 & $\begin{array}{l}\text { Trauma: 1; meningo- } \\
\text { encephalitis: } 2\end{array}$ & 4 mos & $\begin{array}{l}\text { VP \& VA } \\
\text { shunts }\end{array}$ & $\begin{array}{l}47 \% \text { of pts showed clinical improvement postop, w/ } 4 \text { pts } \\
\text { having a good or very good improvement \& } 4 \text { pts having } \\
\text { a moderate improvement. }\end{array}$ \\
\hline
\end{tabular}


» CONTINUED FROM PAGE 4

TABLE 1. Evaluation of studies included in the literature review of patients with NPH

\begin{tabular}{|c|c|c|c|c|c|c|}
\hline Authors \& Year & $\begin{array}{l}\text { Study } \\
\text { Design }\end{array}$ & $\begin{array}{l}\text { No. } \\
\text { of Pts } \\
\text { W/ } \\
\text { sNPH }\end{array}$ & Etiology & $\begin{array}{l}\text { Follow-Up } \\
\text { Period }\end{array}$ & $\begin{array}{l}\text { Shunt } \\
\text { Treatment }\end{array}$ & Outcome \\
\hline $\begin{array}{l}\text { Shimoda et al., } \\
1994\end{array}$ & $\begin{array}{l}\text { Retrospec- } \\
\quad \text { tive }\end{array}$ & 22 & $\begin{array}{l}\text { SAH: } 14 \text {; trauma: } 3 \text {; } \\
\text { tumor: } 1 \text {; menin- } \\
\text { goencephalitis: } 1 \text {; } \\
\text { ICH: } 3\end{array}$ & $\begin{array}{l}\text { Not speci- } \\
\quad \text { fied }\end{array}$ & Not specified & 14 shunt-responsive \& 8 shunt-unresponsive cases. \\
\hline $\begin{array}{l}\text { Tsunoda et al., } \\
2002\end{array}$ & $\begin{array}{l}\text { Retrospec- } \\
\text { tive }\end{array}$ & 17 & $\begin{array}{l}\text { SAH: } 7 \text {; trauma: } 3 \text {; } \\
\text { tumor: } 4 ; \mathrm{ICH}: 3\end{array}$ & $\begin{array}{l}\text { Not speci- } \\
\quad \text { fied }\end{array}$ & Not specified & $\begin{array}{l}\text { Improvement was seen in } 17 / 17 \text { sNPH pts. Some improve- } \\
\text { ment occurred in } 11 / 15 \mathrm{iNPH} \text { pts who had CSF shunting. }\end{array}$ \\
\hline $\begin{array}{l}\text { Yamada et al., } \\
1978\end{array}$ & $\begin{array}{l}\text { Not speci- } \\
\quad \text { fied }\end{array}$ & 12 & SAH: 12 & $\begin{array}{l}\text { Not speci- } \\
\text { fied }\end{array}$ & Not specified & NA \\
\hline Hirai et al., 1993 & $\begin{array}{l}\text { Retrospec- } \\
\text { tive }\end{array}$ & 24 & SAH: 24 & $\begin{array}{l}99 \text { wks } \\
\quad(\text { mean) }\end{array}$ & Not specified & $\begin{array}{l}\text { 18/24 pts w/ sNPH after SAH had excellent \& good out- } \\
\text { comes, whereas } 6 \text { pts had fair or poor outcomes. } 7 / 20 \\
\text { pts w/ iNPH had excellent \& good outcomes, whereas } \\
13 / 20 \text { had fair or poor outcomes. }\end{array}$ \\
\hline $\begin{array}{l}\text { Belloni et al., } \\
\quad 1976\end{array}$ & $\begin{array}{l}\text { Retrospec- } \\
\text { tive }\end{array}$ & 19 & $\begin{array}{l}\text { SAH: } 3 \text {; trauma: } 14 ; \\
\text { meningitis: } 2\end{array}$ & 12 mos & VA shunts & $\begin{array}{l}\text { All pts w/ sNPH improved after shunt insertion. The } 3 \text { pts } \\
\text { w/ iNPH did not improve. }\end{array}$ \\
\hline $\begin{array}{l}\text { Gjerris et al., } \\
1987\end{array}$ & $\begin{array}{l}\text { Prospec- } \\
\text { tive }\end{array}$ & 22 & SAH: 22 & $12 \mathrm{mos}$ & $\begin{array}{l}\text { VP \& VA } \\
\text { shunts }\end{array}$ & $\begin{array}{l}17 / 18 \text { pts w/ sNPH after SAH improved after shunt place- } \\
\text { ment. }\end{array}$ \\
\hline $\begin{array}{l}\text { McGovern et al., } \\
2014\end{array}$ & $\begin{array}{l}\text { Retrospec- } \\
\text { tive }\end{array}$ & 19 & $\begin{array}{l}\text { Trauma: } 1 \text {; tumor: } 15 \text {; } \\
\text { other: } 3\end{array}$ & $\begin{array}{l}\text { Median: } \\
\quad 42 \text { mos }\end{array}$ & $\begin{array}{l}\text { VP \& VA } \\
\text { shunts }\end{array}$ & NA \\
\hline $\begin{array}{l}\text { Børgesen \& } \\
\quad \text { Gjerris, } 1982\end{array}$ & $\begin{array}{l}\text { Prospec- } \\
\text { tive }\end{array}$ & 40 & $\begin{array}{l}\text { SAH: } 16 \text {; trauma: } 10 ; \\
\text { meningoencephali- } \\
\text { tis: 8; other: } 6\end{array}$ & $\begin{array}{c}3 \text { mos \& } \\
1 \mathrm{yr}\end{array}$ & $\begin{array}{c}\text { VA shunts } \\
\text { (16 pts } \\
\text { did not } \\
\text { receive } \\
\text { shunts) }\end{array}$ & $\begin{array}{l}70 \% \text { of pts w/ sNPH experienced an effect from CSF } \\
\text { shunting, compared w/ } 52 \% \text { of pts w/ iNPH. }\end{array}$ \\
\hline $\begin{array}{l}\text { Tisell et al., } \\
2006\end{array}$ & $\begin{array}{l}\text { Prospec- } \\
\text { tive }\end{array}$ & 49 & $\begin{array}{l}\text { SAH: } 18 \text {; trauma: } 12 \text {; } \\
\text { meningoencepha- } \\
\text { litis: } 2 ; \text { CVD: } 7 \text {; } \\
\text { other: } 10\end{array}$ & $\begin{array}{l}4.2 \text { yrs } \\
\text { (mean) }\end{array}$ & VP shunts & $\begin{array}{l}\text { Improvement was seen in } 65 \% \text { of pts w/ sNPH \& in } 55 \% \text { of } \\
\text { pts w/ iNPH. }\end{array}$ \\
\hline $\begin{array}{l}\text { Waldemar et al., } \\
\qquad 1993\end{array}$ & $\begin{array}{l}\text { Not speci- } \\
\text { fied }\end{array}$ & 5 & $\begin{array}{l}\text { SAH: } 2 \text {; trauma: } 2 ; \\
\quad \text { CVD: } 1\end{array}$ & $3 \& 6 \mathrm{mos}$ & VP shunts & $11 / 13$ pts (85\%) improved after CSF shunting. \\
\hline $\begin{array}{l}\text { Moretti et al., } \\
1988\end{array}$ & $\begin{array}{l}\text { Not speci- } \\
\quad \text { fied }\end{array}$ & 6 & $\begin{array}{l}\text { SAH: } 1 \text {; trauma: } 4 \text {; } \\
\text { meningoencepha- } \\
\text { litis: } 1\end{array}$ & 4 mos & Not specified & $\begin{array}{l}\text { 3/6 pts w/ sNPH had clinical improvement after CSF shunt- } \\
\text { ing. }\end{array}$ \\
\hline $\begin{array}{l}\text { Tamaki et al., } \\
1984\end{array}$ & $\begin{array}{l}\text { Not speci- } \\
\quad \text { fied }\end{array}$ & 15 & $\begin{array}{l}\text { SAH: } 5 \text {; trauma: } 7 \text {; } \\
\text { tumor: } 1 \text {; meningo- } \\
\text { encephalitis: } 2\end{array}$ & $\begin{array}{l}\text { Not speci- } \\
\quad \text { fied }\end{array}$ & Not specified & $\begin{array}{l}6 \text { pts had an excellent outcome, } 12 \text { pts had a good out- } \\
\text { come, \& } 13 \text { pts had a poor result after CSF shunting. }\end{array}$ \\
\hline Mori et al., 2002 & $\begin{array}{l}\text { Retrospec- } \\
\text { tive }\end{array}$ & 13 & SAH: 13 & $1 \mathrm{mo}$ & VP shunts & $\begin{array}{l}15 \text { pts had excellent \& good outcomes \& } 7 \text { did not. } 6 / 7 \text { pts } \\
\text { w/ sNPH after SAH improved vs } 3 / 15 \text { pts w/ iNPH. }\end{array}$ \\
\hline $\begin{array}{l}\text { Matsuda et al., } \\
\quad 1990\end{array}$ & $\begin{array}{l}\text { Not speci- } \\
\quad \text { fied }\end{array}$ & 12 & $\begin{array}{l}\text { SAH: } 8 \text {; tumor: } 3 \text {; } \\
\text { CVD: } 1\end{array}$ & $\begin{array}{l}\text { Not speci- } \\
\quad \text { fied }\end{array}$ & VP shunts & All pts showed improvement. \\
\hline $\begin{array}{l}\text { Kamiya et al., } \\
1991\end{array}$ & $\begin{array}{l}\text { Not speci- } \\
\quad \text { fied }\end{array}$ & 4 & $\mathrm{SAH}: 4$ & $\begin{array}{l}\text { Not speci- } \\
\text { fied }\end{array}$ & Not specified & All pts w/ sNPH after SAH improved. \\
\hline $\begin{array}{l}\text { Mamo et al., } \\
1987\end{array}$ & $\begin{array}{l}\text { Not speci- } \\
\text { fied }\end{array}$ & 7 & $\begin{array}{l}\text { SAH: 2; tumor: } 3 \text {; } \\
\text { meningoencepha- } \\
\text { litis: } 2\end{array}$ & 4 mos & $\begin{array}{l}\text { VP \& VA } \\
\text { shunts }\end{array}$ & $\begin{array}{l}\text { Excellent outcome in } 6 \text { pts, good outcome in } 9 \text { pts, fair } \\
\text { in } 7 \text { pts, \& poor in } 3 \text { pts. Overall, } 88 \% \text { of cases were } \\
\text { improved. }\end{array}$ \\
\hline $\begin{array}{l}\text { Tullberg et al., } \\
\qquad 2002\end{array}$ & $\begin{array}{l}\text { Not speci- } \\
\quad \text { fied }\end{array}$ & 13 & $\begin{array}{l}\text { SAH: } 5 \text {; trauma: } 3 \text {; } \\
\text { tumor: } 1 \text {; CVD: } 3 \\
\text { aqueductal steno- } \\
\text { sis: } 1\end{array}$ & 3 mos & $\begin{array}{l}\text { VP or ETV }(2 \\
\text { pts) }\end{array}$ & 27 of the NPH pts improved after shunt surgery $(93 \%)$. \\
\hline
\end{tabular}


» CONTINUED FROM PAGE 5

TABLE 1. Evaluation of studies included in the literature review of patients with NPH

\begin{tabular}{|c|c|c|c|c|c|c|}
\hline Authors \& Year & $\begin{array}{l}\text { Study } \\
\text { Design }\end{array}$ & $\begin{array}{l}\text { No. } \\
\text { of Pts } \\
\text { w/ } \\
\text { sNPH }\end{array}$ & Etiology & $\begin{array}{l}\text { Follow-Up } \\
\text { Period }\end{array}$ & $\begin{array}{l}\text { Shunt } \\
\text { Treatment }\end{array}$ & Outcome \\
\hline $\begin{array}{l}\text { Tullberg et al., } \\
2004\end{array}$ & $\begin{array}{l}\text { Not speci- } \\
\quad \text { fied }\end{array}$ & 19 & $\begin{array}{l}\text { SAH: } 6 \text {; trauma: } 1 \text {; } \\
\text { tumor: } 2 \text {; menin- } \\
\text { goencephalitis: } 1 \text {; } \\
\text { CVD: } 9\end{array}$ & 3 mos & $\begin{array}{l}\text { VP or ETV (2 } \\
\text { pts) }\end{array}$ & $\begin{array}{l}21 / 28 \text { pts improved after shunt surgery in motor \& neuro- } \\
\text { psychological performance }(75 \%), 5 \text { were unchanged, \& } \\
2 \text { pts deteriorated. }\end{array}$ \\
\hline $\begin{array}{l}\text { Tanaka et al., } \\
1997\end{array}$ & $\begin{array}{l}\text { Not speci- } \\
\text { fied }\end{array}$ & 15 & $\begin{array}{l}\text { SAH: } 11 \text {; trauma: } 2 \\
\text { CVD: } 2\end{array}$ & $\begin{array}{l}\text { Not speci- } \\
\text { fied }\end{array}$ & VP shunts & $\begin{array}{l}9 \text { pts improved clinically after CSF shunting, } 12 \text { pts did not } \\
\text { improve. }\end{array}$ \\
\hline $\begin{array}{l}\text { Mase et al., } \\
1998\end{array}$ & $\begin{array}{l}\text { Not speci- } \\
\text { fied }\end{array}$ & 17 & SAH: 17 & $\begin{array}{l}\text { Not speci- } \\
\text { fied }\end{array}$ & Not specified & Not specified. \\
\hline $\begin{array}{l}\text { Kiefer et al, } \\
2006\end{array}$ & $\begin{array}{l}\text { Prospec- } \\
\quad \text { tive }\end{array}$ & 19 & Not specified & $\begin{array}{l}1 \& 12 \\
\text { mos }\end{array}$ & Not specified & $\begin{array}{l}\text { NPH pts responded to shunt surgery in } 71 \% \text { of cases }(66 \% \\
\text { of iNPH pts \& } 82 \% \text { of sNPH pts). }\end{array}$ \\
\hline $\begin{array}{l}\text { Ojemann et al., } \\
1969\end{array}$ & $\begin{array}{l}\text { Retrospec- } \\
\text { tive }\end{array}$ & 10 & $\begin{array}{l}\text { SAH: } 4 \text {; trauma: } 4 \text {; } \\
\text { tumor: } 2\end{array}$ & $6-12$ mos & $\begin{array}{l}\text { Ventriculo- } \\
\text { venous } \\
\text { shunt }\end{array}$ & All pts w/ sNPH had improvement. \\
\hline $\begin{array}{l}\text { Marmarou et al., } \\
\quad 1996\end{array}$ & $\begin{array}{l}\text { Not speci- } \\
\text { fied }\end{array}$ & 7 & Trauma: 7 & $\begin{array}{l}3,6, \& 12 \\
\operatorname{mos}\end{array}$ & Not specified & $\begin{array}{l}\text { Favorable outcome was recorded in } 33.3 \% \text { of pts w/ post- } \\
\text { traumatic hydrocephalus. }\end{array}$ \\
\hline $\begin{array}{l}\text { Hartmann \& } \\
\quad \text { Alberti, } 1977\end{array}$ & $\begin{array}{l}\text { Not speci- } \\
\text { fied }\end{array}$ & 15 & $\begin{array}{l}\text { SAH: } 9 \text {; trauma: } 2 \text {; } \\
\text { tumor: } 2 \text {; menin- } \\
\text { goencephalitis: } 2 \text {; } \\
\text { other: } 1\end{array}$ & $\begin{array}{l}\text { Not speci- } \\
\quad \text { fied }\end{array}$ & VA shunts & Not specified. \\
\hline $\begin{array}{l}\text { Meyer et al., } \\
1985\end{array}$ & $\begin{array}{l}\text { Prospec- } \\
\text { tive }\end{array}$ & 4 & $\begin{array}{l}\text { SAH: } 2 \text {; tumor: } 1 \text {; aq- } \\
\text { ueductal stenosis: } 1\end{array}$ & 4-12 mos & VP shunts & Marked improvement was found in $85 \%$ of cases. \\
\hline $\begin{array}{l}\text { Sahuquillo et al., } \\
1991\end{array}$ & $\begin{array}{l}\text { Prospec- } \\
\text { tive }\end{array}$ & 38 & $\begin{array}{l}\text { SAH: } 12 \text {; trauma: } 8 \text {; } \\
\text { tumor: } 2 \text {; menin- } \\
\text { goencephalitis: } 1 \text {; } \\
\text { aqueductal steno- } \\
\text { sis: } 12 \text {; postcrani- } \\
\text { otomy: } 3\end{array}$ & $\begin{array}{c}6,9,12 \\
\& 24 \\
\text { mos }\end{array}$ & Not specified & $54 / 65$ pts $(83 \%)$ improved. \\
\hline $\begin{array}{l}\text { Soelberg } \\
\text { Sørensen et } \\
\text { al., } 1986\end{array}$ & $\begin{array}{l}\text { Prospec- } \\
\text { tive }\end{array}$ & 5 & $\begin{array}{l}\text { SAH: } 2 \text {; trauma: } 1 \\
\text { meningoencephali- } \\
\text { tis: } 1 \text {; CVD: } 1\end{array}$ & $3-6 \mathrm{mos}$ & $\begin{array}{r}\text { VP or VA } \\
\text { shunts }\end{array}$ & Improvement in $12 / 16$ pts. \\
\hline $\begin{array}{l}\text { Vassilouthis, } \\
1984\end{array}$ & $\begin{array}{l}\text { Retrospec- } \\
\text { tive }\end{array}$ & 24 & $\begin{array}{l}\text { SAH: } 10 \text {; trauma: } 5 \text {; } \\
\text { tumor: } 6 \text {; meningo- } \\
\text { encephalitis: } 3\end{array}$ & $\begin{array}{c}3 \text { mos-4 } \\
\text { yrs }\end{array}$ & VP shunts & $\begin{array}{l}\text { Of } 10 \text { pts w/ SAH, } 9 \text { had excellent improvement \& } 1 \text { had } \\
\text { fair improvement. Of } 6 \text { pts w/ craniotomy for tumor } \\
\text { resection, } 5 \text { had an excellent outcome \& } 1 \text { had a good } \\
\text { outcome. All } 3 \text { pts w/ CSF infection had an excellent } \\
\text { outcome. Of } 5 \text { pts w/ head trauma, } 3 \text { had excellent out- } \\
\text { come, } 1 \text { had a good outcome, \& } 1 \text { had a fair outcome. }\end{array}$ \\
\hline $\begin{array}{l}\text { Piechnik \& } \\
\text { Hultin, } 2005\end{array}$ & $\begin{array}{l}\text { Not speci- } \\
\quad \text { fied }\end{array}$ & 6 & $\begin{array}{l}\text { Trauma: } 3 \text {; CVD: } 2 \text {; } \\
\text { other: } 1\end{array}$ & 3 mos & Not specified & $\begin{array}{l}1 \text { pt worsened, } 7 \text { remained the same, \& } 5 \text { improved after } \\
\text { surgery. }\end{array}$ \\
\hline
\end{tabular}

$\mathrm{CVA}=$ cerebrovascular accident; $\mathrm{ETV}=$ endoscopic third ventriculostomy; $\mathrm{NA}=$ not available; pts = patients.

frontal horns divided by the transverse inner diameter of the cranium) and a "normal" CSF opening pressure measured by lumbar puncture in the lateral recumbent position (normal indicated a CSF pressure as low as $<150 \mathrm{~mm}$ $\mathrm{H}_{2} \mathrm{O}$ or as high as $<240 \mathrm{~mm} \mathrm{H}_{2} \mathrm{O}$ ). Additional diagnostic studies that were used to identify patients with sNPH included MRI in 14 studies and pneumoencephalography in 9 studies. In an attempt to differentiate patients who would benefit the most from shunting (patients with shunt-responsive NPH) from patients who would have less favorable outcomes (shunt-unresponsive patients), a multitude of prognostic tests were performed. The CSF tap test was used in 12 studies, cisternography was performed in 19 studies, cerebral blood flow determination was used in 20 studies, external lumbar drainage was performed in 4 studies, continuous ICP monitoring prior to shunt placement was used in 10 studies, and CSF dynamic studies with determination of conductance and resistance to CSF outflow using a lumbar infusion test were used in 24 studies. 
TABLE 2. Etiologies of sNPH in 1208 patients

\begin{tabular}{ll}
\hline \multicolumn{1}{c}{ Etiology } & No. (\%) \\
\hline SAH & $562(46.5)$ \\
\hline Head trauma & $349(29)$ \\
\hline Brain tumors \& surgery for resection & $75(6.2)$ \\
\hline Meningoencephalitis & $61(5)$ \\
\hline CVD & $55(4.5)$ \\
\hline ICH & $49(4)$ \\
\hline $\begin{array}{l}\text { Other (intracranial ops, radiosurgery, aque- } \\
\text { ductal stenosis, \& Paget's disease) }\end{array}$ & $57(5)$ \\
\hline
\end{tabular}

\section{Treatment of sNPH}

Ventriculoperitoneal (VP) shunting was used to treat patients with sNPH in 25 studies. Seven studies reported treatment with ventriculoatrial (VA) shunting, 10 studies reported that either VA or VP shunting was performed, and 2 studies reported that either VP or lumboperitoneal (LP) shunting was performed to manage sNPH cases. One study used LP shunting and another study used ventriculovenous shunting as the only treatment strategies. The treatment that was used to specifically manage SNPH was reported in 711 patients; $71.9 \%$ had insertion of a VP shunt, $24.4 \%$ had placement of a VA shunt, and $3.7 \%$ were treated with an LP shunt.

Three studies reported the use of a low-pressure valve system, 13 studies reported the use of a medium-pressure valve system, 11 studies used either a low- or a mediumpressure valve system, and 11 studies reported the use of programmable valves. The valve system specifically used in the management of sNPH was reported in 692 patients. Programmable valves were used in $47.8 \%$, medium-pressure valves were used in $39.6 \%$, and a low-pressure valve system was used in $12.6 \%$.

\section{Outcome of NPH}

Several studies combined the outcomes of patients with iNPH and those with sNPH after shunt placement, and could not be included in the outcome analysis. Outcomes were available in 708 patients with sNPH who underwent shunt placement (Table 3). Of these patients, 527 showed clinical improvement (74.4\%); excellent clinical improvement was reported in 58\% of patients. The mean follow-up period after shunt placement was 13 months. In studies that further reported the outcome for the different etiologies of sNPH, $84.2 \%$ of patients with NPH secondary to SAH and $83 \%$ of patients with NPH secondary to head trauma had clinical improvement after shunting. A positive outcome after shunting was reported in $86.4 \%$ of patients with NPH secondary to brain tumors, in $75 \%$ of patients with NPH following meningoencephalitis, in $71.4 \%$ with NPH secondary to ICH, and in $64.7 \%$ of patients with NPH secondary to stroke and CVD. It is important to note that some of the studies that reported the lowest rates of improvement after shunting did not differentiate outcome based on etiology. The overall complication rate after shunt placement was $22 \%$.
TABLE 3. Outcome of sNPH in 708 patients after shunt treatment

\begin{tabular}{lc}
\hline \multicolumn{1}{c}{ Etiology } & \% Clinical Improvement After CSF Shunting \\
\hline SAH & 84.2 \\
\hline Head trauma & 83 \\
\hline Brain tumors & 86.4 \\
\hline Meningoencephalitis & 75 \\
\hline CVD & 64.7 \\
\hline ICH & 71.4 \\
\hline All sNPH cases & 74.4 \\
\hline
\end{tabular}

\section{Discussion}

In the 5 decades since the description of the syndrome of $\mathrm{NPH}$, the understanding of the pathophysiological mechanism is still not fully elucidated, but our knowledge regarding the etiologies, diagnosis, prognosis, treatment, and assessment of patients who will benefit from shunt placement has evolved. Marmarou et al. reported that the "mixing" of cases of iNPH with cases of sNPH has led to considerable controversy regarding diagnostic and therapeutic strategies. ${ }^{35}$ The differentiation between iNPH and sNPH has been highlighted by several authors as well, at least with regard to outcome after shunting..$^{4,30,71}$ Most of the recent work has focused on iNPH, whereas most of what we know regarding SNPH stems from studies originating from the 1960s to the 1990s. Differences in outcomes of iNPH and sNPH have been observed, with substantial improvement after shunting occurring in approximately $30 \%-50 \%$ of patients with no identifiable cause and in about $50 \%-70 \%$ of patients with $\mathrm{SNPH} .{ }^{72}$

The leading cause of sNPH (46.5\%) was SAH. Chronic hydrocephalus develops in approximately $7 \%-37 \%$ of patients with SAH. ${ }^{10}$ Fibrosis and adhesion at the level of the basal cisterns and the subarachnoid and arachnoid villi in patients with SAH can lead to the development of NPH. ${ }^{25}$ Intracranial malignancies $(6.2 \%)$ release proteinaceous products and cellular components into the CSF, which increases CSF viscosity and impairs its reabsorption at the level of the arachnoid granulations, leading to NPH. ${ }^{51}$ There has been a wide variation in the incidence of posttraumatic hydrocephalus, mainly because of the different definitions used to identify this entity. The rate has been reported to be as low as $1 \%$ and as high as $29 \% .{ }^{36}$ Posttraumatic NPH accounted for $29 \%$ of sNPH cases. Similarly, posttraumatic NPH develops when there is impairment in the flow and absorption of CSF. Most etiologies lead to the development of sNPH as a result of 2 mechanisms. The first results from increased content of cells or proteins in the CSF (from SAH, meningitis, tumors, and so on), which causes clogging of CSF outflow and leads to obstruction from reduced absorption through the arachnoid granulations. This results in a decrease in conductance and an increase in resistance to CSF outflow. The second mechanism is due to leptomeningeal fibrosis, arachnoid adhesions, and scarring in the basal cisterns, leading to disturbances in CSF dynamics. ${ }^{5,53}$ There is an initial increase in CSF pressure that results in enlargement of the ventricles. The pressure normalizes afterward 
but the ventricular enlargement is maintained because of Laplace's law. ${ }^{17}$

For NPH to be considered secondary, patients should have a well-established event preceding the occurrence of symptoms that is directly correlated with the development of the characteristic NPH symptoms and imaging findings. The time required for the development of NPH after SAH, trauma, meningitis, stroke, tumors, or intracranial surgery has not been established, but studies have suggested that symptoms may begin immediately after the inciting event, or there may be a delayed onset of a few months' duration. ${ }^{80}$ As described in Wood et al., Hammes et al. reported that obliteration of subarachnoid spaces secondary to fibrosis begins as early as the 10th day after SAH. Ojemann et al. reported that 3 weeks may be sufficient for the development of NPH. ${ }^{46}$ Marmarou et al. reported that hydrocephalus occurred within 2 weeks posttrauma in most patients, and within 1 month in all patients. ${ }^{36}$ Wood et al. reported on 46 cases of sNPH and found that symptoms began either immediately or as late as 4 months after the event, with the majority of patients experiencing onset of disease within 1 month. ${ }^{80}$ Hirai et al. reported that the mean preoperative period was significantly longer in iNPH (10 months vs 1 month). ${ }^{21}$ Other studies have reported that NPH can still develop years after the initial event. ${ }^{2,30}$ In general, most patients develop NPH soon after the primary diseases. Onset of iNPH is usually gradual, with progressive worsening over time. Onset of sNPH may actually be acute or subacute. It is important to determine the time of onset because symptom duration affects the outcome of intervention. ${ }^{12}$

Patients with sNPH do not always show the typical symptoms of dementia, gait abnormalities, and urinary incontinence as in the idiopathic counterpart, and may manifest other atypical symptoms, including seizures, altered consciousness, and motor and sensory deficits. This is because the primary diseases $(\mathrm{SAH}$, traumatic brain injury, intracranial tumors, meningitis, stroke, and so on) often cause severe neurological deficits that might mask the typical NPH symptoms and make it more difficult to establish a diagnosis. ${ }^{77}$ In certain cases, NPH might be suspected only when preexisting neurological deficits begin to deteriorate or when patients fail to have a satisfactory recovery following the initial event. Early diagnosis and shunt placement is essential because it may improve functional outcome and prevent further functional deterioration. Otherwise, functional recovery may be suboptimal. ${ }^{10}$

Several tests have been proposed to accurately identify patients with NPH who will have the best outcome after shunting. These investigations include CT, MRI, isotope cisternography, pneumoencephalography, CSF withdrawal tests, cerebral blood flow measurements, continuous ICP monitoring, and infusion studies with evaluation of CSF dynamics. Whereas the importance of these preoperative evaluations has been widely established in the diagnosis of iNPH, the value of some of these tests remains questionable for sNPH. Stein and Langfitt reported that $80 \%$ of patients with sNPH had significant improvement, whereas significant improvement was only seen in $24 \%$ of iNPH cases. They reported that shunting should be performed directly in patients with $\mathrm{sNPH}$ and that preoperative tests are of little help in selecting this group of patients for surgery. ${ }^{61}$ Børgesen and Gjerris identified a subset of patients with NPH who have a known etiology, short duration of symptoms, severe dementia and gait disturbances, and urinary incontinence who would benefit the most from shunt placement. They reported that these patients are most likely to improve without the need for excessive preoperative testing. ${ }^{4,5}$

On the other hand, patients not fulfilling these criteria should be investigated using other preoperative tests. Wikkelsö et al. reported that NPH after SAH has a good prognosis, and that these patients present typical changes on CT and radionuclide cisternography and represent a minor diagnostic problem as compared with patients with iNPH. ${ }^{79}$ Gjerris et al. reported that CSF dynamic studies can be used to predict outcomes in patients with $\mathrm{sNPH}$ after SAH, especially in patients with high resistance to CSF outflow. ${ }^{14}$ Similarly, Marmarou et al. suggested that CSF dynamic studies can help in establishing the diagnosis and prognosis of patients with sNPH that results from head trauma. ${ }^{36}$ When patients display atypical symptoms of NPH, CSF dynamic studies can help in deciding whether to perform shunt placement for those with suspected sNPH. ${ }^{78}$

Responsiveness to shunting procedures constitutes the most important difference between idiopathic and secondary forms of NPH. Of the patients with sNPH, 74.4\% were found to have improvement in clinical status after shunting. Børgesen reported that of 31 patients without a known etiology, only $42 \%$ improved 1 year after shunting, whereas $72 \%$ of patients with a known etiology had improved. The trend toward better results in patients with a known etiology was significant $(\mathrm{p}<0.01) .{ }^{4}$ In the same study it was reported that both patients with iNPH and those with sNPH had similar pretreatment clinical status, and that the observed difference in outcome cannot be attributed to differences in the initial clinical status. Vanneste et al. reported that marked improvement occurred in $52 \%$ of patients with sNPH, lowering to $15 \%$ when only patients with iNPH were considered..$^{70}$ They further conducted a literature review of 1047 patients in which they showed that studies in patients with iNPH found marked improvement in $33 \%$, whereas studies with mixed patients found marked improvement in $50 \%$. They concluded that the etiology of NPH has a significant effect on outcome. Larsson et al. reported that patients with sNPH had a better prognosis than did those with iNPH (94\% SAH, 79\% posttraumatic, $77 \%$ idiopathic). ${ }^{30}$

The duration of preoperative symptoms has been reported to be a significant factor in the prognosis of NPH. ${ }^{16,30}$ Patients with SNPH present with a shorter duration of preoperative symptoms, because the presence of a known etiology can lead to an earlier investigation and detection of NPH symptoms. Similarly, Thomsen et al. reported that patients with NPH of a known cause had a better outcome than patients with iNPH, and that disease duration of less than 12 months was an important factor for successful outcome. They reported that patients with sNPH usually have a more abrupt onset of dementia, whereas patients with iNPH usually experience gradual deterioration. ${ }^{65}$ Wood et al. reported that symptom duration of less than 6 months is 
a good prognostic factor for improvement after shunting. However, their study was one of the few to conclude that etiology did not have an important prognostic value. ${ }^{80}$

Other studies have further suggested that patients with sNPH may have a more favorable shunt outcome than patients with iNPH, because patients with iNPH may have brain atrophy in addition to hydrocephalic features, whereas sNPH patients only have a CSF circulatory disorder. ${ }^{64,67}$ Although our results support the separation of iNPH and sNPH based on outcome, iNPH and sNPH do not represent separate entities; rather, they represent a clinical syndrome associated with a subset of patients with acquired hydrocephalus-patients of different ages with different etiologies and vastly different pathophysiology. Both iNPH and SNPH represent a disorder of CSF flow and absorption, although iNPH may be associated with other factors including white matter ischemic changes, increased transmantle pressure, asymptomatic fibrosing meningitis, and insufficiency of the transcortical subarachnoid space. ${ }^{20,37}$

Both VP and VA shunting have been used successfully in the management of sNPH. The most important factor to consider in the treatment of patients with sNPH is early intervention. Wen et al. reported that shunt placement should be performed within 6 months of the onset of posttraumatic hydrocephalus. ${ }^{78}$ Some studies that used programmable valves observed a higher rate of reprogramming in patients with iNPH. Zemack and Romner reported that in the iNPH group $49 \%$ of patients required valve adjustments, versus $32.4 \%$ of patients with sNPH (because of overdrainage, underdrainage, and subdural hematoma). ${ }^{83}$ Similarly, Yamashita et al. reported that shunt reprogramming was more frequently performed in patients with iNPH than in patients with sNPH after SAH. ${ }^{82}$ The best response to treatment was observed in the $\mathrm{SAH}$, head trauma, and intracranial malignancy groups. These patients may benefit the most from shunt surgery for sNPH.

Patients with sNPH who require surgical shunt placement are often being treated with anticoagulation and antiplatelet agents to manage the primary disease, including stroke or endovascular interventions for aneurysm treatment in patients with SAH, or these patients may be on anticoagulation therapy for the management of other comorbid conditions (atrial fibrillation, deep venous thrombosis, valvular heart disease, and so on). Whether shunting should be performed in these patients is controversial. ${ }^{13}$ The benefits-to-risk ratio should be assessed for each individual. The feared complications of shunting in patients on anticoagulation therapy include $\mathrm{ICH}$, subdural hematoma, abdominal bleeding, and thromboembolic complications after discontinuation of antithrombotic agents. Goodwin et al. reported a low rate of subdural hematoma (6.7\%) in patients on long-term warfarin therapy, and they observed no thromboembolic complications when they stopped warfarin prior to shuting..$^{15}$ They concluded that anticoagulation was not a contraindication for shunting in patients with NPH. Perioperative management of antithrombotic therapy can be accomplished safely, and anticoagulation treatment should not prevent the placement of a shunt in patients with sNPH.

The combination of several factors supports the existence of sNPH, including the facts that sNPH can occur in any age group, versus iNPH, which occurs most commonly in the elderly population; that an acute or subacute onset is associated with sNPH following the suspected etiology, versus a slow, gradual onset in patients with $\mathrm{iNPH}$; that typical symptoms of NPH may not always be present with sNPH and atypical symptoms may occur; and that there are higher rates of improvement after shunt placement in patients with sNPH compared with iNPH, which plays an important role in patient selection for shunting. Although we evaluated the various SNPH etiologies, further separation of cases based on etiology is not clearly supported from our data and requires further studies.

The limitations of this review relate to paucity of the literature on the topic of sNPH; the search strategy with the inclusion of smaller studies and older studies; and the inclusion of heterogeneous studies that have a wide variation in the size, population, methodology (both prospective and retrospective studies), and outcome definition. In addition, the lack of randomization of the included studies, with each one having its own reporting bias, should be highlighted as well. It is important to assess the likely extent of the bias and its potential impact on the conclusions. Nevertheless, for this rarely discussed subject, Class I or II evidence is difficult to obtain. This review paper evaluates patients with one clinical pattern of acquired hydrocephalus (NPH) and cannot be generalized to the entire acquired hydrocephalus population. Because of the limitations of this review, further studies are recommended.

\section{Conclusions}

Secondary NPH does indeed exist and should be differentiated from iNPH based on outcome as well as clinical, pathophysiological, and epidemiological characteristics but should not be considered as a separate entity. Evaluation of patients with NPH to identify a known cause is recommended because the response to treatment varies considerably. Although clinical presentation is often the same, a multitude of primary etiologies can lead to the development of sNPH. The most common etiologies of sNPH include SAH, traumatic brain injury, intracranial malignancies, meningitis, and stroke. Further studies are required to investigate differences in management and outcome among the diverse etiologies of sNPH.

\section{References}

1. Adams RD, Fisher CM, Hakim S, Ojemann RG, Sweet WH: Symptomatic occult hydrocephalus with "normal" cerebrospinal-fluid pressure. A treatable syndrome. N Engl J Med 273:117-126, 1965

2. Belloni G, di Rocco C, Focacci C, Galli G, Maira G, Rossi GF: Surgical indications in normotensive hydrocephalus. A retrospective analysis of the relations of some diagnostic findings to the results of surgical treatment. Acta Neurochir (Wien) 33:1-21, 1976

3. Bergsneider M, Black PM, Klinge P, Marmarou A, Relkin $\mathrm{N}$ : Surgical management of idiopathic normal-pressure hydrocephalus. Neurosurgery 57 (3 Suppl):S29-S39, ii-v, 2005

4. Børgesen SE: Conductance to outflow of CSF in normal pressure hydrocephalus. Acta Neurochir (Wien) 71:1-45, 1984

5. Børgesen SE, Gjerris F: The predictive value of conductance 
to outflow of CSF in normal pressure hydrocephalus. Brain 105:65-86, 1982

6. Bradley WG: Normal pressure hydrocephalus: new concepts on etiology and diagnosis. AJNR Am J Neuroradiol 21:1586-1590, 2000

7. Chang CC, Kuwana N, Ito S: Management of patients with normal-pressure hydrocephalus by using lumboperitoneal shunt system with the Codman Hakim programmable valve. Neurosurg Focus 7(4):e8, 1999

8. Chang CC, Kuwana N, Ito S, Ikegami T: Prediction of effectiveness of shunting in patients with normal pressure hydrocephalus by cerebral blood flow measurement and computed tomography cisternography. Neurol Med Chir (Tokyo) 39:841-846, 1999

9. Chen IH, Huang CI, Liu HC, Chen KK: Effectiveness of shunting in patients with normal pressure hydrocephalus predicted by temporary, controlled-resistance, continuous lumbar drainage: a pilot study. J Neurol Neurosurg Psychiatry 57:1430-1432, 1994

10. Chen Z, Song W, Du J, Li G, Yang Y, Ling F: Rehabilitation of patients with chronic normal-pressure hydrocephalus after aneurysmal subarachnoid hemorrhage benefits from ventriculoperitoneal shunt. Top Stroke Rehabil 16:330-338, 2009

11. Eide PK, Sorteberg W: Changes in intracranial pulse pressure amplitudes after shunt implantation and adjustment of shunt valve opening pressure in normal pressure hydrocephalus. Acta Neurochir (Wien) 150:1141-1147, 2008

12. Finney GR: Normal pressure hydrocephalus. Int Rev Neurobiol 84:263-281, 2009

13. Gelabert-González M, Iglesias-Pais M, García-Allut A, Martínez-Rumbo R: Chronic subdural haematoma: surgical treatment and outcome in 1000 cases. Clin Neurol Neurosurg 107:223-229, 2005

14. Gjerris F, Børgesen SE, Sørensen PS, Boesen F, Schmidt K, Harmsen A, et al: Resistance to cerebrospinal fluid outflow and intracranial pressure in patients with hydrocephalus after subarachnoid haemorrhage. Acta Neurochir (Wien) 88:7986, 1987

15. Goodwin CR, Kharkar S, Wang P, Pujari S, Rigamonti D, Williams MA: Evaluation and treatment of patients with suspected normal pressure hydrocephalus on long-term warfarin anticoagulation therapy. Neurosurgery 60:497502, 2007

16. Gustafson L, Hagberg B: Recovery in hydrocephalic dementia after shunt operation. J Neurol Neurosurg Psychiatry 41:940-947, 1978

17. Hakim S, Adams RD: The special clinical problem of symptomatic hydrocephalus with normal cerebrospinal fluid pressure. Observations on cerebrospinal fluid hydrodynamics. J Neurol Sci 2:307-327, 1965

18. Hartmann A, Alberti E: Differentiation of communicating hydrocephalus and presenile dementia by continuous recording of cerebrospinal fluid pressure. J Neurol Neurosurg Psychiatry 40:630-640, 1977

19. Hashimoto K, Shibasaki H, Tabuchi K: Auditory brainstem responses before and after shunting in patients with suspected normal pressure hydrocephalus. Neurol Med Chir (Tokyo) 30:29-35, 1990

20. Hebb AO, Cusimano MD: Idiopathic normal pressure hydrocephalus: a systematic review of diagnosis and outcome. Neurosurgery 49:1166-1186, 2001

21. Hirai O, Kikuchi H, Ishikawa M: Idiopathic vs. postSAH normal pressure hydrocephalus, in Avezaat CJJ, van Eijndhoven JHM, Maas AIR, et al (eds): Intracranial Pressure VIII. Berlin: Springer, 1993, pp 842-844

22. Ishikawa M, Kikuchi H, Taki W, Kobayashi A, Nishizawa $\mathrm{S}$, Yonekura Y, et al: Regional cerebral blood flow and oxygen metabolism in normal pressure hydrocephalus after subarachnoid hemorrhage. Neurol Med Chir (Tokyo) 29:382-388, 1989

23. Kahlon B, Sundbärg G, Rehncrona S: Comparison between the lumbar infusion and CSF tap tests to predict outcome after shunt surgery in suspected normal pressure hydrocephalus. J Neurol Neurosurg Psychiatry 73:721-726, 2002

24. Kamiya K, Yamashita N, Nagai H, Mizawa I: Investigation of normal pressure hydrocephalus by ${ }^{123}$ I-IMP SPECT. Neurol Med Chir (Tokyo) 31:503-507, 1991

25. Kibler RF, Couch RS, Crompton MR: Hydrocephalus in the adult following spontaneous subarachnoid haemorrhage. Brain 84:45-61, 1961

26. Kiefer M, Eymann R, Steudel WI: Outcome predictors for normal-pressure hydrocephalus. Acta Neurochir Suppl 96:364-367, 2006

27. Kilic K, Czorny A, Auque J, Berkman Z: Predicting the outcome of shunt surgery in normal pressure hydrocephalus. J Clin Neurosci 14:729-736, 2007

28. Klinge P, Marmarou A, Bergsneider M, Relkin N, Black PM: Outcome of shunting in idiopathic normal-pressure hydrocephalus and the value of outcome assessment in shunted patients. Neurosurgery 57 (3 Suppl):S40-S52, ii-v, 2005

29. Kosteljanetz M, Nehen AM, Kaalund J: Cerebrospinal fluid outflow resistance measurements in the selection of patients for shunt surgery in the normal pressure hydrocephalus syndrome. A controlled trial. Acta Neurochir (Wien) 104:48-53, 1990

30. Larsson A, Wikkelsö C, Bilting M, Stephensen H: Clinical parameters in 74 consecutive patients shunt operated for normal pressure hydrocephalus. Acta Neurol Scand 84:475482, 1991

31. Lee JH, Park DH, Back DB, Lee JY, Lee CI, Park KJ, et al: Comparison of cerebrospinal fluid biomarkers between idiopathic normal pressure hydrocephalus and subarachnoid hemorrhage-induced chronic hydrocephalus: a pilot study. Med Sci Monit 18:PR19-PR25, 2012

32. Magnaes B: Communicating hydrocephalus in adults. Diagnostic tests and results of treatment with medium pressure shunts. Neurology 28:478-484, 1978

33. Mamo HL, Meric PC, Ponsin JC, Rey AC, Luft AG, Seylaz JA: Cerebral blood flow in normal pressure hydrocephalus. Stroke 18:1074-1080, 1987

34. Marmarou A, Bergsneider M, Klinge P, Relkin N, Black PM: The value of supplemental prognostic tests for the preoperative assessment of idiopathic normal-pressure hydrocephalus. Neurosurgery 57 (3 Suppl):S17-S28, ii-v, 2005

35. Marmarou A, Bergsneider M, Relkin N, Klinge P, Black PM: Development of guidelines for idiopathic normalpressure hydrocephalus: introduction. Neurosurgery 57 (3 Suppl):S1-S3, ii-v, 2005

36. Marmarou A, Foda MA, Bandoh K, Yoshihara M, Yamamoto T, Tsuji O, et al: Posttraumatic ventriculomegaly: hydrocephalus or atrophy? A new approach for diagnosis using CSF dynamics. J Neurosurg 85:1026-1035, 1996

37. Marmarou A, Young HF, Aygok GA: Estimated incidence of normal pressure hydrocephalus and shunt outcome in patients residing in assisted-living and extended-care facilities. Neurosurg Focus 22(4):E1, 2007

38. Mase M, Yamada K, Banno T, Miyachi T, Ohara S, Matsumoto T: Quantitative analysis of CSF flow dynamics using MRI in normal pressure hydrocephalus. Acta Neurochir Suppl 71:350-353, 1998

39. Mathew NT, Meyer JS, Hartmann A, Ott EO: Abnormal cerebrospinal fluid-blood flow dynamics. Implications in diagnosis, treatment, and prognosis in normal pressure hydrocephalus. Arch Neurol 32:657-664, 1975 
40. Matsuda M, Nakasu S, Nakazawa T, Handa J: Cerebral hemodynamics in patients with normal pressure hydrocephalus: correlation between cerebral circulation time and dementia. Surg Neurol 34:396-401, 1990

41. McGovern RA, Kelly KM, Chan AK, Morrissey NJ, McKhann GM II: Should ventriculoatrial shunting be the procedure of choice for normal-pressure hydrocephalus? J Neurosurg 120:1458-1464, 2014

42. McQuarrie IG, Saint-Louis L, Scherer PB: Treatment of normal pressure hydrocephalus with low versus medium pressure cerebrospinal fluid shunts. Neurosurgery 15:484488, 1984

43. Meyer JS, Kitagawa Y, Tanahashi N, Tachibana H, Kandula P, Cech DA, et al: Evaluation of treatment of normal-pressure hydrocephalus. J Neurosurg 62:513-521, 1985

44. Moretti JL, Sergent A, Louarn F, Rancurel G, le Percq M, Flavigny R, et al: Cortical perfusion assessment with 123I-isopropyl amphetamine (123I-IAMP) in normal pressure hydrocephalus (NPH). Eur J Nucl Med 14:73-79, 1988

45. Mori K, Maeda M, Asegawa S, Iwata J: Quantitative local cerebral blood flow change after cerebrospinal fluid removal in patients with normal pressure hydrocephalus measured by a double injection method with $\mathrm{N}$-isopropyl-p-[ $\left.{ }^{123} \mathrm{I}\right]$ iodoamphetamine. Acta Neurochir (Wien) 144:255-263, 2002

46. Ojemann RG, Fisher CM, Adams RD, Sweet WH, New PF: Further experience with the syndrome of "normal" pressure hydrocephalus. J Neurosurg 31:279-294, 1969

47. Owler BK, Momjian S, Czosnyka Z, Czosnyka M, Péna A, Harris NG, et al: Normal pressure hydrocephalus and cerebral blood flow: a PET study of baseline values. J Cereb Blood Flow Metab 24:17-23, 2004

48. Panagiotopoulos V, Konstantinou D, Kalogeropoulos A, Maraziotis T: The predictive value of external continuous lumbar drainage, with cerebrospinal fluid outflow controlled by medium pressure valve, in normal pressure hydrocephalus. Acta Neurochir (Wien) 147:953-958, 2005

49. Pfisterer WK, Aboul-Enein F, Gebhart E, Graf M, Aichholzer M, Mühlbauer M: Continuous intraventricular pressure monitoring for diagnosis of normal-pressure hydrocephalus. Acta Neurochir (Wien) 149:983-990, 2007

50. Piechnik SK, Hultin L: Postoperative changes in SPECTrCBF in hydrocephalus. Acta Neurochir Suppl 95:169-172, 2005

51. Pirouzmand F, Tator CH, Rutka J: Management of hydrocephalus associated with vestibular schwannoma and other cerebellopontine angle tumors. Neurosurgery 48:1246-1254, 2001

52. Poca MA, Mataró M, Matarín M, Arikan F, Junqué C, Sahuquillo J: Good outcome in patients with normal-pressure hydrocephalus and factors indicating poor prognosis. J Neurosurg 103:455-463, 2005

53. Rangel-Castilla L, Barber S, Zhang YJ: The role of endoscopic third ventriculostomy in the treatment of communicating hydrocephalus. World Neurosurg 77:555560,2012

54. Reinprecht A, Czech T, Dietrich W: Clinical experience with a new pressure-adjustable shunt valve. Acta Neurochir (Wien) 134:119-124, 1995

55. Relkin N, Marmarou A, Klinge P, Bergsneider M, Black PM: Diagnosing idiopathic normal-pressure hydrocephalus. Neurosurgery 57 (3 Suppl):S4-S16, ii-v, 2005

56. Sahuquillo J, Rubio E, Codina A, Molins A, Guitart JM, Poca MA, et al: Reappraisal of the intracranial pressure and cerebrospinal fluid dynamics in patients with the so-called "normal pressure hydrocephalus" syndrome. Acta Neurochir (Wien) 112:50-61, 1991

57. Salmon JH: Adult hydrocephalus. Evaluation of shunt therapy in 80 patients. J Neurosurg 37:423-428, 1972
58. Shiino A, Nishida Y, Yasuda H, Suzuki M, Matsuda M, Inubushi T: Magnetic resonance spectroscopic determination of a neuronal and axonal marker in white matter predicts reversibility of deficits in secondary normal pressure hydrocephalus. J Neurol Neurosurg Psychiatry 75:11411148,2004

59. Shimoda M, Oda S, Shibata M, Masuko A, Sato O: Change in regional cerebral blood flow following glycerol administration predicts. Clinical result from shunting in normal pressure hydrocephalus. Acta Neurochir (Wien) 129:171-176, 1994

60. Soelberg Sørensen P, Jansen EC, Gjerris F: Motor disturbances in normal-pressure hydrocephalus. Special reference to stance and gait. Arch Neurol 43:34-38, 1986

61. Stein SC, Langfitt TW: Normal-pressure hydrocephalus. Predicting the results of cerebrospinal fluid shunting. J Neurosurg 41:463-470, 1974

62. Tamaki N, Kusunoki T, Wakabayashi T, Matsumoto S: Cerebral hemodynamics in normal-pressure hydrocephalus. Evaluation by ${ }^{133} \mathrm{Xe}$ inhalation method and dynamic CT study. J Neurosurg 61:510-514, 1984

63. Tanaka A, Kimura M, Nakayama Y, Yoshinaga S, Tomonaga M: Cerebral blood flow and autoregulation in normal pressure hydrocephalus. Neurosurgery 40:1161-1167, 1997

64. Tarnaris A, Kitchen ND, Watkins LD: Noninvasive biomarkers in normal pressure hydrocephalus: evidence for the role of neuroimaging. J Neurosurg 110:837-851, 2009

65. Thomsen AM, Børgesen SE, Bruhn P, Gjerris F: Prognosis of dementia in normal-pressure hydrocephalus after a shunt operation. Ann Neurol 20:304-310, 1986

66. Tisell M, Hellström P, Ahl-Börjesson G, Barrows G, Blomsterwall E, Tullberg M, et al: Long-term outcome in 109 adult patients operated on for hydrocephalus. Br J Neurosurg 20:214-221, 2006

67. Tsunoda A, Mitsuoka H, Bandai H, Endo T, Arai H, Sato $\mathrm{K}$ : Intracranial cerebrospinal fluid measurement studies in suspected idiopathic normal pressure hydrocephalus, secondary normal pressure hydrocephalus, and brain atrophy. J Neurol Neurosurg Psychiatry 73:552-555, 2002

68. Tullberg M, Hellström P, Piechnik SK, Starmark JE, Wikkelsö C: Impaired wakefulness is associated with reduced anterior cingulate $\mathrm{CBF}$ in patients with normal pressure hydrocephalus. Acta Neurol Scand 110:322-330, 2004

69. Tullberg M, Hultin L, Ekholm S, Månsson JE, Fredman P, Wikkels $\varnothing \mathrm{C}$ : White matter changes in normal pressure hydrocephalus and Binswanger disease: specificity, predictive value and correlations to axonal degeneration and demyelination. Acta Neurol Scand 105:417-426, 2002

70. Vanneste J, Augustijn P, Dirven C, Tan WF, Goedhart ZD: Shunting normal-pressure hydrocephalus: do the benefits outweigh the risks? A multicenter study and literature review. Neurology 42:54-59, 1992

71. Vanneste J, Augustijn P, Tan WF, Dirven C: Shunting normal pressure hydrocephalus: the predictive value of combined clinical and CT data. J Neurol Neurosurg Psychiatry 56:251-256, 1993

72. Vanneste JA: Diagnosis and management of normal-pressure hydrocephalus. J Neurol 247:5-14, 2000

73. Vassilouthis J: The syndrome of normal-pressure hydrocephalus. J Neurosurg 61:501-509, 1984

74. Vorstrup S, Christensen J, Gjerris F, Sørensen PS, Thomsen AM, Paulson OB: Cerebral blood flow in patients with normal-pressure hydrocephalus before and after shunting. J Neurosurg 66:379-387, 1987

75. Walchenbach R, Geiger E, Thomeer RT, Vanneste JA: The value of temporary external lumbar CSF drainage in predicting the outcome of shunting on normal pressure hydrocephalus. J Neurol Neurosurg Psychiatry 72:503506,2002 
76. Waldemar G, Schmidt JF, Delecluse F, Andersen AR, Gjerris F, Paulson OB: High resolution SPECT with [99mTc]-d,1HMPAO in normal pressure hydrocephalus before and after shunt operation. J Neurol Neurosurg Psychiatry 56:655664, 1993

77. Wang C, Du HG, Yin LC, He M, Zhang GJ, Tian Y, et al: Analysis of related factors affecting prognosis of shunt surgery in patients with secondary normal pressure hydrocephalus. Chin J Traumatol 16:221-224, 2013

78. Wen L, Wan S, Zhan RY, Li G, Gong JB, Liu WG, et al: Shunt implantation in a special sub-group of post-traumatic hydrocephalus-patients have normal intracranial pressure without clinical representations of hydrocephalus. Brain Inj 23:61-64, 2009

79. Wikkelsö C, Andersson H, Blomstrand C, Lindqvist G, Svendsen P: Normal pressure hydrocephalus. Predictive value of the cerebrospinal fluid tap-test. Acta Neurol Scand 73:566-573, 1986

80. Wood JH, Bartlet D, James AE Jr, Udvarhelyi GB: Normalpressure hydrocephalus: diagnosis and patient selection for shunt surgery. Neurology 24:517-526, 1974

81. Yamada F, Fukuda S, Samejima H, Yoshii N, Kudo T: Significance of pathognomonic features of normalpressure hydrocephalus on computerized tomography. Neuroradiology 16:212-213, 1978

82. Yamashita N, Kamiya K, Yamada K: Experience with a programmable valve shunt system. J Neurosurg 91:26-31, 1999

83. Zemack G, Romner B: Adjustable valves in normal-pressure hydrocephalus: a retrospective study of 218 patients.

Neurosurgery 51:1392-1402, 2002

\section{Disclosures}

Dr. Tjoumakaris is a consultant for Medtronic and Stryker.

\section{Author Contributions}

Conception and design: all authors. Acquisition of data: Daou, Klinge. Analysis and interpretation of data: Jabbour, Daou, Tjoumakaris. Drafting the article: Jabbour, Daou. Critically revising the article: Jabbour, Daou, Tjoumakaris. Reviewed submitted version of manuscript: Jabbour, Daou, Tjoumakaris, Rosenwasser. Approved the final version of the manuscript on behalf of all authors: Jabbour. Administrative/technical/material support: Rosenwasser. Study supervision: Jabbour, Klinge, Rosenwasser.

\section{Correspondence}

Pascal M. Jabbour, Division of Neurovascular Surgery and Endovascular Neurosurgery, Department of Neurological Surgery, Thomas Jefferson University Hospital, 901 Walnut St., 3rd Fl., Philadelphia, PA 19107. email: pascal.jabbour@jefferson.edu. 